\title{
A NEW METHOD FOR IMPROVING SCATTEROMETER WIND QUALITY CONTROL
}

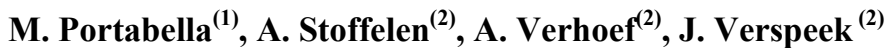 \\ (1) Unidad de Tecnología Marina - CSIC, Pg. Marítim Barceloneta 37-49, 08003 Barcelona, Spain \\ (2) Royal Netherlands Meteorological Institute (KNMI), Postbus 201, 3730 AE De Bilt, The Netherlands
}

Email:portabella@,cmima.csic.es

\begin{abstract}
An important part of the scatterometer wind data processing is the quality control (QC). This paper shows the implementation of a new scatterometer QC procedure, based on a comprehensive analysis of the wind inversion residual, which significantly improves the effectiveness of the wind data QC. The method is applied on the Advanced Scatterometer (ASCAT) onboard Metop-A, but is generic and can therefore be applied to any scatterometer system.
\end{abstract}

\section{INTRODUCTION}

Scatterometers are satellite-based real aperture radar instruments known to provide accurate mesoscale (25$50 \mathrm{~km}$ resolution) sea surface wind field information used in a wide variety of applications, including Numerical Weather Prediction (NWP) data assimilation, nowcasting, and climate studies. The radar antenna geometry, the measurement noise, as well as nonlinearities in the relationship between the backscatter measurements in a Wind Vector Cell (WVC) and the mean wind vector complicate the wind retrieval process. In addition, scatterometers are sensitive to geophysical phenomena other than WVC-mean wind, such as local wind variability, confused sea state, rain, and land \& ice contamination of the radar footprint. These phenomena can distort the wind signal, leading to poor-quality retrieved winds. As such, elimination of poor quality data is a prerequisite for the successful use of scatterometer winds.

The Metop-A satellite was launched on 19 October 2006 and carries the Advanced Scatterometer (ASCAT). The radar operates at $\mathrm{C}$ band and is vertically polarized, with three fan beam antennas pointing at each side of the sub-satellite track. An important tool in the interpretation of the ASCAT data is the visualization of triplets of radar backscatter or $\sigma^{\circ}$ measurements (corresponding to the three antenna beams) in the 3dimensional measurement space at each cross-track WVC [1]. For such given WVC position, the ASCAT measured triplets are distributed around a well-defined "conical" surface (see Figure 1) and hence the signal largely depends on just two geophysical parameters, i.e., wind speed and direction, since geometrical aspects are rather constant over the orbit. Such cone consists of the Geophysical Model Function (GMF), which represents the best fit to the measured triplets. The magnitude of the triplet departures from the two-parameter function (i.e., the GMF) is correlated with the quality of the wind retrievals [1]. A way to investigate such departures is to look at the inversion residual or maximum likelihood estimator (MLE) parameter, which can be interpreted as a measure of the distance between the set of radar measurements (triplets) and the cone surface in a slightly transformed measurement space.

In general, the triplets lie close to the cone surface (i.e., low MLE values), further validating the two-parameter (i.e., wind-vector dependent) GMF. As shown by several QC procedures developed for previous scatterometer missions (e.g., [2]), a large inconsistency with the GMF results in a large absolute MLE, which indicates geophysical conditions other than those modelled by the GMF, such as local wind variability, rain, confused sea state, or ice, and as such, the MLE provides a good indication for the quality of the retrieved winds. However, no work has been done to investigate the correlation between the quality of the retrieved winds and the MLE sign, i.e., positive (negative) for triplets located inside (outside) the cone surface.

In this paper, we propose a QC method, which not only depends on the magnitude of the MLE but also on its sign. In Section 2, the ASCAT operational QC is introduced. In Section 3, the MLE sign computation is presented. The analysis of the MLE sign in terms of a wind quality indicator is described in Section 4. Finally, it is concluded in Section 5 that the MLE sign is very beneficial for QC.

\section{ASCAT OPERATIONAL WIND QUALITY CONTROL}

The most common approach used for scatterometer wind inversion is the Bayesian approach, which leads to the Maximum Likelihood Estimator (MLE) technique [1], [3], [4], [5]. For the ERS and ASCAT scatterometers, the following simplified MLE function is minimized [1]:

$M L E=\frac{1}{3} \sum_{i=1}^{3}\left(z_{o i}-z_{s i}\right)^{2}$

where $i$ is the measurement index, $z_{o i}=\left(\sigma_{o i}^{o}\right)^{1.6}$ is the transformed backscatter measurement, and $z_{s i}=\left(\sigma_{s i}^{o}\right)^{1.6}$ is the transformed backscatter simulated through the 
GMF. The C-band GMF, i.e., the so-called CMOD5n [6], is represented in z-space in the following way:

$$
z_{s}(\theta, v, \phi)=B_{0}(\theta, v)^{0.625}\left[1+B_{1}(\theta, v) \cos \phi+B_{2}(\theta, v) \cos (2 \phi)\right]
$$

where $\theta, \phi$, and $v$ are the incidence angle, azimuthal wind direction angle and wind speed, respectively. $B_{0}$, $B_{1}$, and $B_{2}$ are functions of wind speed and incidence angle and were obtained by fitting the GMF to ERS scatterometer data which covers an incidence angle range of $18-55^{\circ}$.

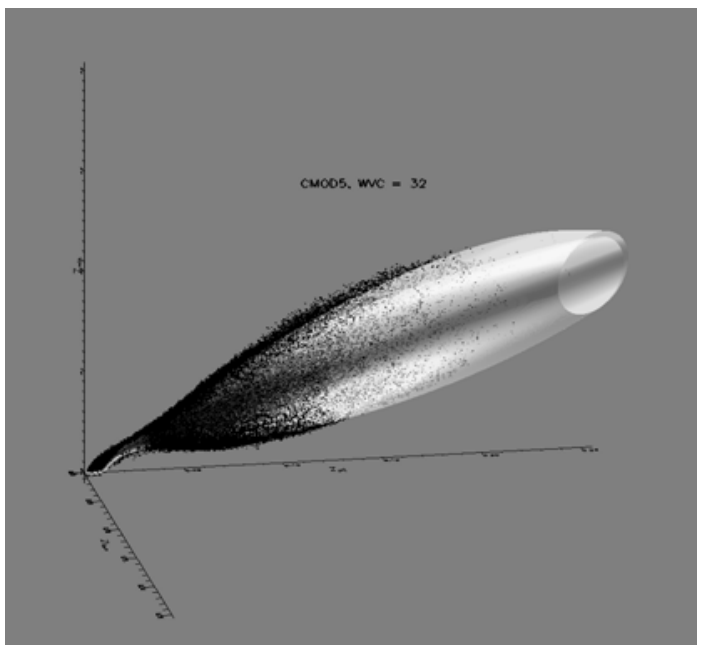

Figure 1. Visualization of the CMOD5n GMF (grey surface) and the ASCAT triplets (black dots) in $3 D$ measurement space, for WVC number 32. The axes represent the fore-, aft- and mid beam backscatter in $z$ space, i.e., $\left(z_{\text {fore }}, z_{\text {aft }}, z_{\text {mid }}\right)$ where $z=\left(\sigma^{0}\right)^{0.625}$.

Different wind speed and direction trial values are used in the GMF in order to minimize the MLE (Eq. 1). Neglecting the GMF uncertainty, the following equation is used: $z_{o i}=\left(\sigma_{s i}^{o}+\varepsilon_{i}\right)^{1.6}$, where $\varepsilon_{i}$ is the backscatter error with relative error $K p_{i}=\varepsilon_{i} / \sigma_{s i}^{o}$, to determine the expectation value of Eq. 1 by using a Taylor expansion:

$<M L E>=\frac{1}{3} \sum_{i=1}^{3}\left(1.6 \cdot z_{s i} \cdot K p_{i}\right)^{2}$

The MLE inversion technique is also used for other scatterometers, albeit using a slightly different formulation [3] and therefore different expectation value [2]. The expected MLE (Eq. 3) is very valuable for QC [1], [2] and wind retrieval purposes [7], [8].

Detailed information on the estimation of measurement errors (Kp) can be found in [9]. Several studies [1], [2], [9] show that $\mathrm{Kp}$ misestimation leads to differences in the expected MLE across the sub-satellite track. As such, an additional empirically-derived WVCdependent normalization is generally derived for QC and wind retrieval purposes [2], [10]. In summary, scatterometer wind QC is generally based on the MLE normalized by the estimated measurement noise through a WVC-dependent factor.
For ASCAT, the QC procedure implemented in the operational Level 2 ASCAT Wind Data Processor (AWDP) during the first three years of the MetOp-A mission, was also based on the normalized MLE, similar to that of the ERS scatterometer [9]. AWDPv1.0.18 provides two wind solutions. The inversion residual or MLE was normalized by the measurement noise [9] and a WVC-dependent factor. A fixed threshold value of this normalized MLE (hereafter referred to as MLE), i.e., 18.6, is used in AWDP to discriminate between poor and good quality wind data. As such, a WVC with MLE value below (above) 18.6 is accepted (rejected) in the processing chain. Table 1 summarizes the overall performance of the ASCAT MLE-based QC. The mean vector root-mean-square (VRMS) difference between ASCAT and European Centre for Medium-range Forecasts (ECMWF) model winds is used as a quality indicator. The substantial difference in terms of quality (relative to ECMWF model winds) between accepted and rejected WVCs denotes an effective QC.

\begin{tabular}{|c|c|c|}
\hline QC & $\begin{array}{c}\text { Fraction of } \\
\text { data (\%) }\end{array}$ & VRMS (m/s) \\
\hline Accepted & $99.55(99.61)$ & $1.79(1.79)$ \\
\hline Rejected & $0.45(0.39)$ & $5.68(6.12)$ \\
\hline
\end{tabular}

Table 1. Percentage and Mean VRMS difference between ASCAT and ECMWF winds for accepted and rejected data. Note that the scores for the new QC (see sections 3 and 4) are shown in parenthesis.

\section{MLE SIGN}

Figure 2 shows a schematic illustration of a cross section of the cone surface shown in Figure 1. Note that such cross section is almost perpendicular to the cone, and mainly shows the variation due to wind direction $(\varnothing)$ at approximately constant wind speed $(\cos (2 \phi)$ term in Eq. 2 [1]). By computing the inner product of $\vec{H}=\vec{z}_{s}-\vec{A}_{0}$ and $\vec{M}=\vec{z}_{s}-\vec{z}_{o}$ (see Figure 2), one can straightforwardly determine whether the measurement triplet is inside or outside the cone surface, i.e., the inner product will be positive when the triplet $\left(\vec{z}_{0}\right)$ is inside the cone and negative when the triplet is outside the cone.

The triplets on the GMF surface, i.e., $\vec{z}_{s}$, are given by the inversion. The ASCAT minimization procedure (Eq. 1) generally produces 2 wind vector solutions. For each wind solution $\left[v_{s o l}, \phi_{s o l}\right]$, a $\vec{z}_{s}$ can be derived using Eq. 2. Likewise, the cone's major axis location can be derived from Eq. 2, i.e., $\vec{A}_{0}=\vec{B}_{0}\left(\theta, v_{\text {sol }}\right)^{0.625}$.

As such, for each minimum MLE value in Eq. 1, i.e., $M L E_{\text {sol }}$ (inversion residual corresponding to a wind solution), a sign can be added in the following way:

$M L E_{\text {sol }}^{\prime}=\frac{\vec{H} \bullet \vec{M}}{\|\vec{H} \bullet \vec{M}\|} \cdot M L E_{\text {sol }}$ 
To mimic the old (signless) MLE-based procedure with the new parameter MLE' (Eq. 4), the ASCAT QC threshold (18.6) needs to be applied to its absolute value, i.e., |MLE'|.

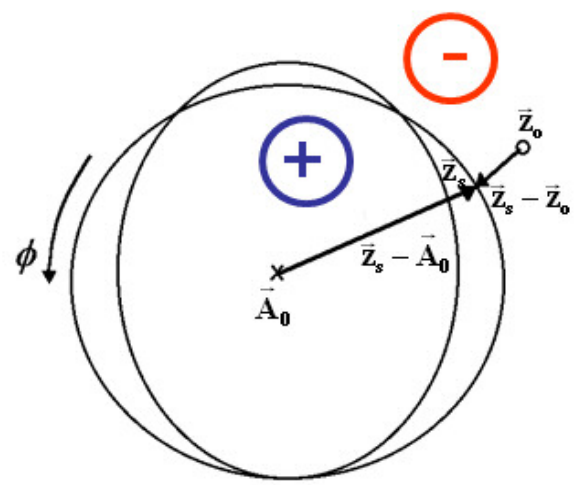

Figure 2. Schematic illustration of a cross section of the CMOD5n GMF shown in Figure 1. Note that $\vec{z}_{s}$ refers

to a point on the cone surface as determined by inversion; $\vec{A}_{0}$ represents the major axis location at this cross section; and $\vec{z}_{0}$ depicts the measurement triplet.

Figure 3 shows a 2-D histogram of the ASCAT retrieved wind speed versus the $1^{\text {st }}$ rank MLE' value (hereafter referred to as MLE'), where the $1^{\text {st }}$ rank solution corresponds to the absolute minimum in Eq. 1, i.e., the closest distance between the ASCAT triplet and the CMOD5n cone surface. A good fit of CMOD5n to the ASCAT measurements is represented by a symmetric MLE' distribution in Fig. 3, i.e., same MLE distribution inside and outside the cone. For mid to high winds this is roughly the case. However, at low winds, a very distinct behaviour is shown. Around $4 \mathrm{~m} / \mathrm{s}$ most of the triplets lie inside the cone, indicating a clear misfit of the CMOD5n GMF. At very low winds (below $2 \mathrm{~m} / \mathrm{s}$ ), we see the opposite behaviour, i.e., most of the triplets lie outside the cone. This is due to the closing of the cone as it approaches its origin, i.e., the origin of the measurement space $\left(z_{\text {fore }}, z_{\text {aft }}, z_{\text {mid }}\right)=(0,0,0)$. By construction the CMOD5n $\mathrm{z}_{\mathrm{si}}$ values for the different beams (incidence angles) will converge at zero winds, which leads the cone cross section (as illustrated in Fig.2) to virtually disappear. At very low winds and 25$50 \mathrm{~km}$ resolution cells, the scatterometer mainly observes wind variability rather than a WVC-mean (close to $0 \mathrm{~m} / \mathrm{s}$ ) vector wind and with little backscatter anisotropy, such that the conical surface closes and no triplets can reside inside the cone (see Figure1), which therefore mostly lie outside the (very small) cone cross section.

Although, due to the effects described above, the scatterometer wind direction skill is low at low winds, the scatterometer wind vector error is about the same at low winds than at moderate and high winds [11]. Moreover, it is most relevant to study the effect of the
MLE sign on QC above $4 \mathrm{~m} / \mathrm{s}$. The analysis in the following section is therefore focused on scatterometer winds above $4 \mathrm{~m} / \mathrm{s}$.

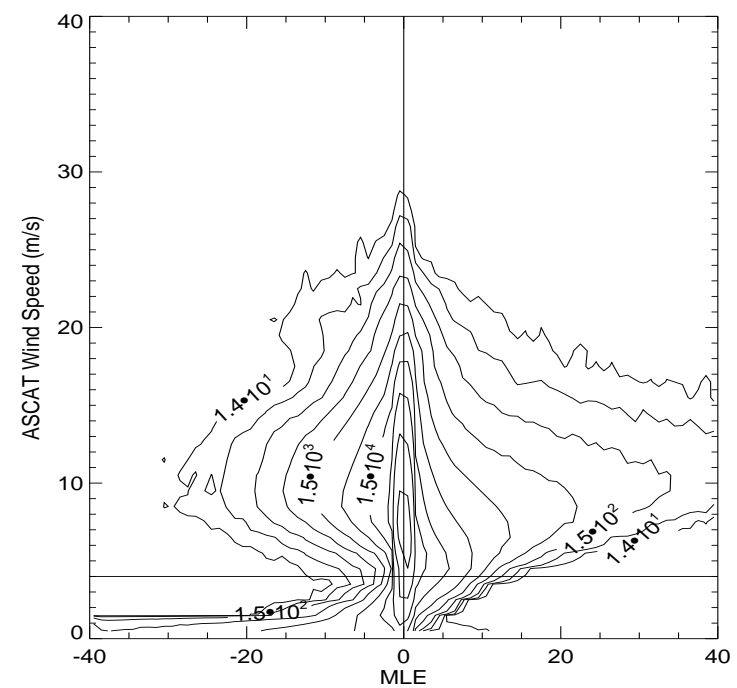

Figure 3. Two-dimensional histogram of the ASCAT retrieved wind speed versus MLE'value for the $1^{\text {st }}$-rank solution (i.e., corresponding to closest distance between the triplet and the cone surface). The contour lines are in logarithmic scale: every two steps is a factor of 10 and the lowest level is at 14 WVCs per bin.

\section{ANALYSIS OF QC WITH MLE SIGN}

To characterize the correlation between the MLE and the quality of retrieved winds, ECMWF winds are used as reference. One year (2008) of collocated ASCAT and ECMWF data are used in the analysis. Figure 4 shows the mean VRMS difference between ASCAT and ECMWF winds as a function of the MLE magnitude and sign, i.e., MLE'. There is a clear distinct behaviour for positive and negative MLE' values in terms of data quality. On the one hand, ASCAT winds derived from triplets located inside the cone rapidly decrease in quality as the triplet's distance to the cone surface increases (i.e., see steep increase of VRMS as a function of MLE', for positive MLE' values). The quality degradation is present in both wind speed and direction components (not shown). On the other hand, for triplets lying outside the cone surface, the ASCAT wind quality degradation is generally small regardless of the triplet distance to the cone (see the relatively small slope of the VRMS curve as a function of MLE', for negative MLE' values). As such, since January 2010, the AWDP QC procedure was updated to account not only for the MLE magnitude but also for the MLE sign. In particular, the new QC procedure does not reject any wind data from triplets located outside the cone surface (i.e., with negative MLE' values). The MLE threshold value is kept the same (18.6), although it is not applied to the absolute value of the MLE' but to the MLE' value itself. As shown in Table 1 (in parenthesis), the new QC 
rejects about $0.39 \%$ of data, i.e., about $12 \%$ less rejections than the old QC. Also note that while the VRMS of accepted data is about the same $(1.79 \mathrm{~m} / \mathrm{s})$, that of rejected data is $0.44 \mathrm{~m} / \mathrm{s}$ larger with the new QC, denoting an improved effectiveness of the latter.

Figure 5 shows the histogram of the wind direction relative to the ASCAT mid beam direction for two different wind sources, i.e., ASCAT (solid) and ECMWF (dotted), and for three different MLE' intervals: $-18.6<$ MLE' $<18.6$ (top), MLE' $>18.6$ (mid), and MLE' $<-18.6$ (bottom). Note that the top (mid) panel represents QC-accepted (QC-rejected) WVCs in both the previous (before January 2010) and the current AWDP QC. The bottom panel shows the WVCs previously rejected and currently accepted.

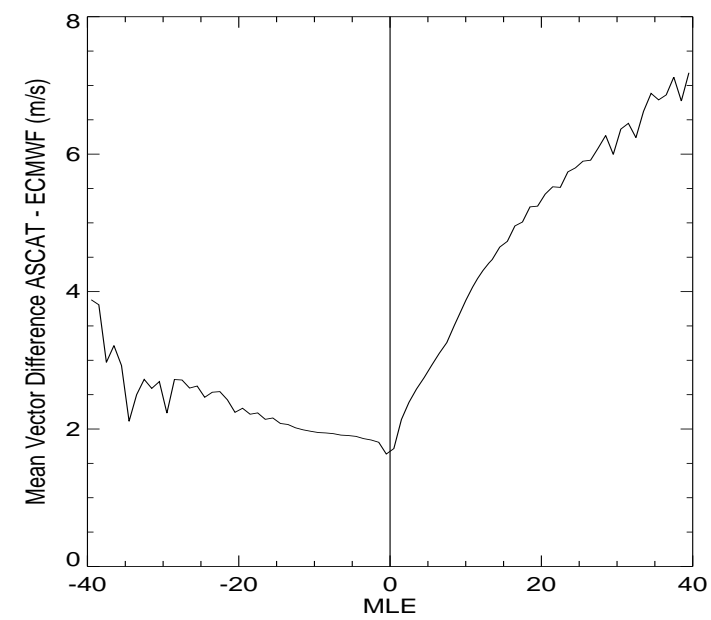

Figure 4. Mean vector root-mean-square (VRMS) difference between ASCAT and ECMWF winds as a function of MLE'. Note that negative (positive) values correspond to triplets outside (inside) the cone surface.

The top and middle panels correspond to the MLE intervals where both old and new QCs coincide. The ASCAT wind direction distribution is very similar to that of ECMWF for the QC-accepted WVCs (top), following the good agreement between both wind sources in Table 1. However, for rejected WVCs (mid), the ASCAT distribution is substantially different from that of ECMWF. The former has clear artificial (non geophysical) accumulations at certain wind directions (see solid curve peaks while the dotted curve is rather flat). This systematic effect in the direction retrievals is well known in scatterometry and has been reported by several authors (e.g., [5], [12]). It is undesirable and therefore important to remove or at least to mitigate. As such, by detecting such artefacts, the QC shows good performance.

Regarding the MLE interval where old and new QCs differ (bottom panel), although ASCAT wind directions present a somewhat more modal distribution than that of ECMWF, it is clear that both distributions are rather similar, indicating a fair ASCAT wind direction skill for MLE' $<-18.6$. This is consistent with the rather small VRMS values shown in Fig. 4 at such MLE' interval.

An explanation for the different wind direction skill (and therefore wind vector skill) for triplets located far inside and far outside the cone surface is the following. Let's assume, for example, a true wind with a crosswind direction (relative to mid beam), which can be represented in Fig. 2 by a point at the bottom of the cross section. Take a measurement triplet $\left(\vec{z}_{0}\right)$ inside the cone and close to the true wind direction and move it away from the surface. At a certain point, the triplet may lie closer to the opposite side of the cross section, i.e., the top part (upwind/downwind directions), therefore leading to a set of very wrong wind directions in the retrieval process. Another interesting effect is that when the triplet lies close to the centre of the cross section, the number of retrieved wind direction ambiguities increases from two (typical case for ASCAT) to four (not shown). Now, take the same crosswind triplet, but this time located outside the cone, and move it down away from the surface. The triplet's closest distance to the cone will remain in the crosswind region, indicating that the wind direction skill is not much affected in this case. Moreover, the number of retrieved ambiguities remains the same, i.e., two (not shown). Similarly, fore or aft noise contributions cause larger wind direction errors for triplets within the cone than for triplets outside the cone (not shown).

\section{CONCLUSIONS}

The ASCAT QC is improved by taking into account the MLE sign in addition to the MLE magnitude. It is found that for triplets lying outside the cone, the ASCAT retrieved winds are generally of good quality, as compared to ECMWF winds. For ASCAT triplets located far away from the cone surface (MLE > 18.6), the wind direction skill is found to be poor when triplets are inside the cone (MLE'> 18.6) and reasonably good when the triplets are outside the cone (MLE' < -18.6). As such, the AWDP QC has been updated in January 2010 to also account for the sign of the MLE value. This method is generic and opens the grounds for a more sophisticated QC for both past and future scatterometer missions.

The MLE analysis is also relevant in the context of a GMF improvement. A good GMF fit of the backscatter measurements should result in approximately the same amount of measurement triplets inside and outside the GMF cone surface (except for very low winds). An analysis of the current C-band GMF (CMOD5n), reveals that for winds around $4 \mathrm{~m} / \mathrm{s}$, the majority of triplets lie inside the cone, therefore indicating a GMF misfit (see section 3). Furthermore, spatial patterns (i.e., maps) of the MLE sign are found to be correlated with sub-cell wind variability, indicating the potential 
sensitivity of scatterometer systems to the presence of, e.g., wind gustiness.
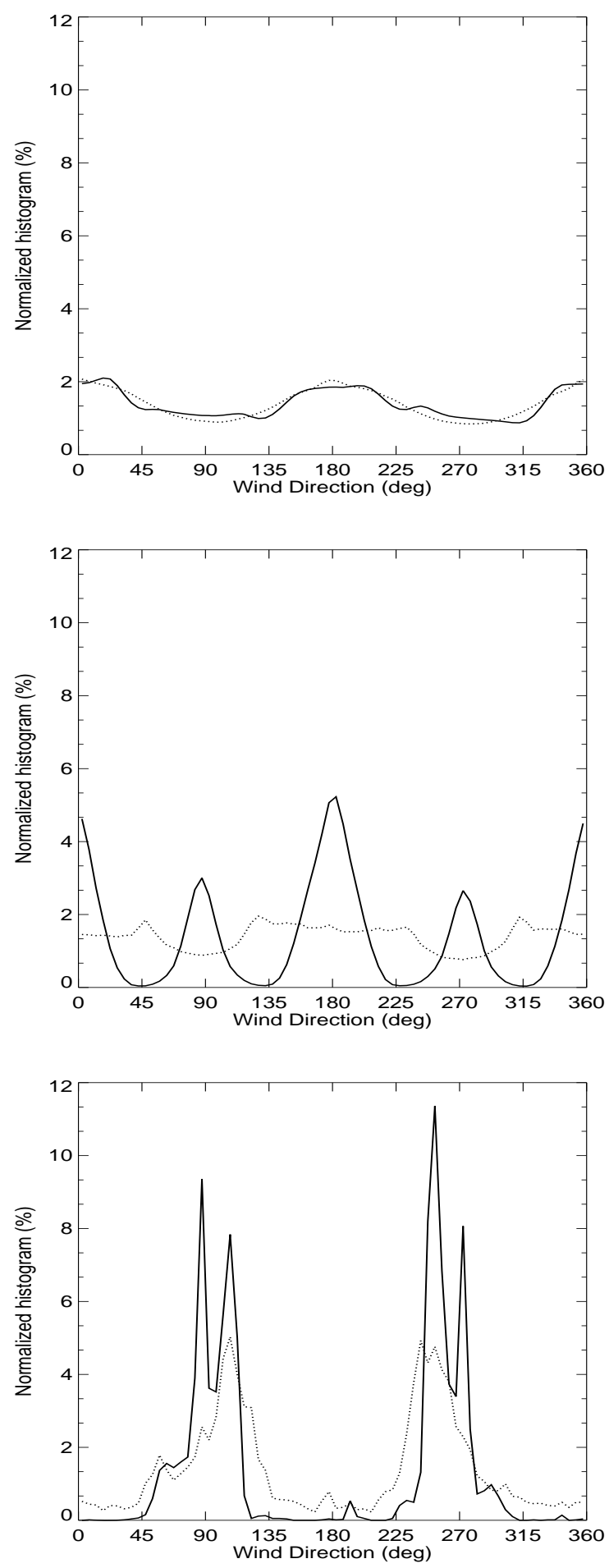

Figure 5. Wind direction (with respect to the ASCAT mid beam azimuth) histograms for ASCAT (solid) and ECMWF (dotted) winds for $-18.6<M L E^{\prime}<18$ (top), $18<M L E^{\prime}<40$ (middle) and $-40<M L E^{\prime}<-18$ (bottom).

\section{ACKNOWLEDGEMENTS}

The work has been funded under the EUMETSAT Ocean and Sea Ice (OSI) Satellite Application Facility (SAF) Associated Scientist project reference CDOPSG06-VS03. The ASCAT level 1b data are provided by EUMETSAT. The software used in this work has been developed through the EUMETSAT Numerical Weather Prediction SAF. The authors would also like to thank the two reviewers who helped to improve the paper.

\section{REFERENCES}

[1] Stoffelen, A., and Anderson, D., "Scatterometer data interpretation: measurement space and inversion," $J$. Atmos. and Oceanic Technol. 14 (6), 1298-1313, 1997.

[2] Portabella, M., and Stoffelen, A. (2001). Rain detection and quality control of SeaWinds. J. Atm. and Ocean Techn., 18 (7), pp. 1171-1183.

[3] Pierson, W.J., "Probabilities and statistics for backscatter estimates obtained by a scatterometer," $J$. Geophys. Res., vol. 94, no. C7, pp. 9743-9759, 1989.

[4] Cornford, D., Csató, L., Evans, D. J., and Opper, M., "Baysian analysis of the scatterometer wind retrieval inverse problems: some new approaches," J. R. Statist. Soc. B, vol. 66, no. 3, pp. 609-626, 2004.

[5] Stoffelen, A., and Portabella, M. (2006). On Bayesian scatterometer wind inversion. IEEE Trans. Geosci. Rem. Sens., 44 (6), doi:10.1109/TGRS.2005.862502, pp. 1523-1533.

[6] Hersbach, H., A. Stoffelen, and S. de Haan (2007). The improved C-band ocean geophysical model function: CMOD-5. J. Geophys. Res., 112, C03006, doi:10.1029/2006JC003743.

[7] Stiles, B.W., Pollard, B.D., Dunbar, R.S., "Direction interval retrieval with thresholded nudging," IEEE Trans. Geosci. Rem. Sens. 40 (1), pp. 79-89, 2002.

[8] Portabella, M., and Stoffelen, A., "A probabilistic approach for SeaWinds data assimilation," Quart. J. R. Met. Soc., vol. 130, no. 596, pp. 127-152, 2004.

[9] Portabella, M., and Stoffelen, A., "Scatterometer backscatter uncertainty due to wind variability," IEEE Trans. Geosci. Rem. Sens., 44 (11), doi:10.1109/TGRS.2006.877952, pp. 3356-3362, 2006.

[10] Huddleston, J.N., and Stiles, B.W., "A Multidimensional Histogram Technique for Flagging Rain Contamination on QuikSCAT," Proc. of IEEE Internat. Geoscience and Remote Sensing Symposium, Vol. 3, Honolulu (USA), IEEE, pp. 1232-1234, 2000.

[11] Stoffelen, A., 'Error modeling and calibration: towards the true surface wind speed," J. Geophys. Res., vol. 103, no. C4, pp. 7755-7766, 1998.

[12] Ebuchi, N., and Graber, H. C. (1998). Directivity of wind vectors derived from the ERS-1/AMI scatterometer. J. Geophys. Res., vol. 103(C4), pp. 77877798 . 